\title{
Hemophagocytic lymphohistiocytosis associated with HBV-HCV coinfection in adult: case report
}

\author{
mounia bendari ${ }^{1}$, nouama bouanani ${ }^{2}$, hanane delsa $^{3}$, rajaa jabbouri ${ }^{4}$, sara nejjari ${ }^{3}$, safaa \\ darouich $^{3}$, and kamal doghmi ${ }^{3}$ \\ ${ }^{1}$ Mohammed VI University of Health Sciences (UM6SS) \\ ${ }^{2}$ Mohammed VI University of Health Sciences, Casablanca, Morocco \\ ${ }^{3} 1$ - Mohammed VI University of Health Sciences, Casablanca, Morocco \\ ${ }^{4}$ Affiliation not available
}

May 18, 2020

\begin{abstract}
Hemophagocytic syndrome have experienced a very significant revival in recent years, we report a rare case of secondary HLH to Hepatitis $\mathrm{B}$ virus (HBV) and hepatitis $\mathrm{C}$ virus (HCV) coinfection. In addition to the treatment for hepatitis the patient underwent therapy for HLH, with this treatment we obtained complete remission.
\end{abstract}

\section{Key clinical messages}

The case reported is the first case in Morocco to our knowledge

The reason for sharing this case is to facilitate knowledge transfer between physicians, caring for adult patients with HLH, with the aim to improve the outcome of these patients.

\section{Introduction :}

Hemophagocytic syndromes (HS) have experienced a very significant revival in recent years, there are considered as a rare pathology but a lot of progress has been made thanks to improved knowledge and the multiplication of publications and the establishment of well-coded protocol for treating this syndrome.

HS have poor diagnosis, it can be definded by overstimulation of the immune system leading to systemic inflammation, cytokine storm and multi-organ failure. ${ }^{1}$

They constitute a heterogeneous group and can be divided into two primary hemophagocyte categories lymphohistiocytosis (HLH) and secondary hemophagocytic syndromes. ${ }^{2}$

Herein we report a rare case of a woman with stage secondary HLH to Hepatitis B virus (HBV) and hepatitis $\mathrm{C}$ virus (HCV) coinfection, the first case in Morocco to our knowledge. The patient achieved complete remission with antiviral treatment and chemotherapy. Through this work we highlight the need of more research and collaboration across all physicians as well as sharing information about similarities and differences between cases, and about their management to improve the overall prognosis and to raise awareness about this malignancy among physicians and patients.

\section{Case report:}

We report the rare observation about young woman, aged 47 years old, she was initially referred to our hospital from Central Africa with complaints of few weeks of epigastralgia with anaemic syndrome and 
deterioration of the general state and persistent fever. The initial physical examination findings revealed fever of $40^{\circ} \mathrm{C}$ without signs of infection, pulse $115 / \mathrm{min}$, respiratory rate $20 / \mathrm{min}$, and blood pressure 110/60 $\mathrm{mmHg}$, pallor with frank cutaneous and mucosal jaundice as well as abdominal distension with hepatosplenomegaly, important edema of the lower limbs, Vital signs and neurological exam were normal, and no oxygen was required.

The biological assessment with complete blood count after recent red cell transfusion revealed pancytopenia (haemoglobin was at $5.8 \mathrm{~g} / \mathrm{dl}$, white blood cells counts $2.4 \mathrm{Giga} / \mathrm{L}$, neutrophils level at 1,3 Giga/L, lymphocytes cells count at $0,9 \mathrm{Giga} / \mathrm{L}$, platelets at $10 \mathrm{Giga} / \mathrm{L}$ ), C-Reactive Protein level was at $88 \mathrm{mg} / \mathrm{L}$, procalcitonin was negative, hypoalbuminemia at $24 \mathrm{~g} / \mathrm{l}$, the research of tuberculosis infections was done and turned out negative. The rest of the infectious workup has demonstrated the absence of a toxoplasmosis or leishmaniasis infection, blood cultures were negatives, the rate of Lactic Acidosis was to 2241UI/L.

The assessment showed also slight cytolysis, an abdominal ultrasound showed signs of portal hypertension (splenomegaly, dilated portal vein) and chronic liver disease.

The upper gastroduodenoscopy revealed gastric vascular ectasia, also known as 'watermelon stomach' without associated esogastric varices. To assess the cause of cirrhosis, the viral serology B and C were performed and confirmed a co-infection of viral hepatitis $\mathrm{B}$ and $\mathrm{C}$. The viral load results from the quantitative HCV-RNA and HBV-DNA in the serum were respectively 5,61 $\log \mathrm{UI} / \mathrm{ml}(407380 \mathrm{UI} / \mathrm{mL})$ and $2.02 \mathrm{log} \mathrm{UI} / \mathrm{ml}(104$ $\mathrm{UI} / \mathrm{mL})$.

Given the persistence of the fever and the worsening of the general condition of the patient and the negativity of the infectious assessment, a complementary assessment of febrile pancytopenia was carried out and made it possible to highlight the presence of images of hemophagocytosis at the bone marrow aspiration (Figure A), as well as the existence of hyper-ferritinemia at $1535 \mathrm{ng} / \mathrm{ml}$, hypertriglyceridemia at $3.9 \mathrm{~g} / \mathrm{l}$. Given all these clinical and biological criteria, the diagnosis retained was the association of a macrophagic activation syndrome and an HBV-HCV coinfection (codominant) with compensated cirrhosis.

The patient management combined the treatment for HLHs (Etoposide+ dexamethasone) according to the HLH-94 protocol and antiviral therapy for HBV-HCV coinfection with supportive care.

This antiviral therapy was based on the European Association for the Study of the liver (EASL) Guidelines and was adapted to the drugs available in Morocco: Sofosbuvir + Daclatasvir for 6 months for hepatitis C and Tenofovir for hepatitis B.

The evolution was favorable with obtaining apyrexia, the cytopenia was resolved, total disappearance of jaundice, very clear improvement of the general state, regression of transfusion needs until transfusion independence, normalization of the biological balance, with undetectable viral load for both viruses after 3 months of treatment.

\section{Discussion:}

In 1979 Risdall et al. individualized a new entity clinic characterized by fever, hepato-splenomomegaly and pancytopenia. Lymphadenopathy, rash and lung damage were often present. in this first description it was also observed that the bone marrow and the lymph nodes were infiltrated by macrophages of normal morphology, which phagocytized erythrocytes, platelets and leukocytes. ${ }^{3}$

the autopsy of six fatal cases showed subarachnoid and hepatic infiltration with hepatocytic necrosis. It was the first precise description of hemophagocytosis syndrome.

The pathophysiology of HS has become better and better understood in recent years thanks to the study of primary forms. it was recognized that HS is due to a activation and / or cytotoxicity defect of CD8 T cells and NK cells which produce large amounts of $\gamma$ interferon which leads to activation of macrophages in the bone marrow and reticuloendothelial cells which in turn produce proinflammatory cytokine. ${ }^{4}$

Indeed, plasma levels pro-inflammatory cytokines IL-1, IL-6, TNF $\alpha$, IFN $\gamma$ M-CSF and IL-18 were elevated. 
They are roughly divided into primary hemophagocytes lymphohistiocytosis (HLH) and secondary hemophagocytic syndromes. Primary HLH is the consequence of genetic mutations altering the cytotoxic function of the natural killer (NK) and cytotoxic T cells and generally presents in infancy and childhood. in primary HLH we find familial HLH (F HLH), where patients have autosomal recessive mutations affecting Perforin (FHLH2), MUNC 13-4 (UNC13D), STX11 (coding for syntaxin 11), and STXBP2 (coding for syntaxin-binding protein 2). ${ }^{5,6}$

Primary HLH also includes other inherited immunodeficiency syndromes with hypo pigmentation such as Chédiak-Higashi syndrome, Griscelli syndrome, and type II Hermansky-Pudlak syndrome. ${ }^{7}$

In the other hand, we have secondary HLH, they usually affect adolescents and adults but, they are considered as reactive syndrome. Most cases of secondary HLH are associated with "predisposing condition" causing immune dysregulation, such as malignancy (particularly lymphoma), immunodeficiency, or autoimmune disease, and/or a "trigger", most commonly infection such as Epstein-Barr virus (EBV). ${ }^{8}$

In adults, infections represent the most prevalent triggers of HLH, with increasing age, other causes are found like malignancies, primarily lymphomas. ${ }^{9,10}$

$\backslash$ sout

For Concerning our patient, the clinical presentation was concordant with infectious disease, the assessment found of fever and jaundice. the etiological assessment found a viral hepatitis B and C infection, however despite the antiviral treatment the persistence of the fever pushed us to carry out more assessment at the research of HLH which turned out to be positive.

In fact, the diagnosis of HLH in adults must be bone according to the HLH-2004 diagnostic criteria in association with clinical judgment and the patient's history

In 1991, the Histiocyte Society suggests a standardized set of 5 diagnostic criteria for HLH used for the prospective HLH-94 clinical trial. In 2004, a revision for these criteria was proposed and validated, the new revision included more diagnosis criteria, for positif diagnosis of HLH, individuals needed to meet 5 of 8 diagnostic criteria. ${ }^{11,12}$

The positive diagnosis of HLH can be confirmed if a molecular diagnosis consistent with HLH, or presence of 5 of the following criteria: Fever, Splenomegaly, Cytopenias (affecting 2 of 3 lineages in the peripheral blood), Hemoglobin less than $90 \mathrm{~g} / \mathrm{L}$ (hemoglobin less than $100 \mathrm{~g} / \mathrm{L}$ in infants ,4 wk), Platelets less than 100 Giga/L, Neutrophils less than1 Giga/L, Hypertriglyceridemia and/or, hypofibrinogenemia, Fasting triglycerides more than $3.0 \mathrm{mmol} / \mathrm{L}$ (ie, more than $265 \mathrm{mg} / \mathrm{dL}$ ), Fibrinogen more than $1.5 \mathrm{~g} / \mathrm{L}$, Hemophagocytosis in bone marrow or spleen or lymph nodes. No evidence of malignancy., Low or no NK cell activity (according to local laboratory reference), Ferritin more than $500 \mathrm{mg} / \mathrm{L}$, sCD25 (ie, soluble IL-2 receptor) more than 2400 $\mathrm{U} / \mathrm{mL}$

Other diagnosis tools that are not part of the HLH-2004 criteria, consist on hyperbilirubinemia, hepatomegaly, transaminitis (usually found on patients with HLH), and elevated lactate dehydrogenase and D-dimer levels, with the latter elevated on the majority of patient even when international normalized ratio, partial thromboplastin time, and fibrinogen are normal. These futures facilitates disctincting between HLH and septic shock and conditions such as autoimmune hemolytic anemia, and they can help for response evaluation to therapy. ${ }^{13}$

For our patient, criteria for HLH were present, she was febrile, she had a splenomegaly, cytopenia, hyperferritinemia, hypertriglyceridemia, and specific images of hemophagositosis. For this patient exploring the presence of peripheral hemophagocytosis has been proposed quickly for identifying adult secondary HLH because she was admitted to our unit with sepsis and pancytopenia.

The viral hepatitis are among the leading causes of chronic liver disease worldwide and the first cause of cirrhosis in Africa. However, The Hepatitis B virus (HBV) and hepatitis C virus (HCV) coinfection is a complex clinical entity that has an estimated worldwide prevalence of $1-15 \% .{ }^{14}$ Most clinical studies have shown 
that progression of disease is faster in HBV-HCV coinfected patients compared to those with monoinfection, with high rates of decompensated cirrhosis ${ }^{15}$ and increased incidence of Hepatocellular carcinoma. ${ }^{16}$

Treatment in coinfected patients is complex, specially due to interaction of the two viruses.

Until now, there are no clear treatment guidelines for HBV-HCV coinfection. But many study showed good results with the new drugs like direct acting antiviral agents. ${ }^{17}$

These treatment should be discuss for all candidates for chemotherapy and immunosuppressive therapy with active viral hepatitis specially B.

Concerning adults with infection triggered-HLH the first line therapy consists of antimicrobials. In these cases, the role of chemotherapy and immunosuppression is not clear. Sometimes, systemic steroids can be added to antimicrobials but its benefits are unknown due to lack of data. ${ }^{18}$

In our case, in addition to the treatment for hepatitis the patient underwent specific therapy for HLH according to HLH-94 protocol, ${ }^{19}$ with this treatment we obtained the improvement of general condition, transfusion independence, decrease and disparition of splenomegaly.

\section{Conclusion:}

HLH is considered as a fatal disease, despite its low incidence, it should be brought up quickly in the presence of any unexplained prolonged fever.

In our case, HLH was associated with an HCV-HBV coinfection which is very rare. The early concomitant treatment of viral hepatitis infection and HLH saved our patient.

All physicians should be aware of HLH, because early recognition may prevent irreversible organ damage and subsequent death $(14,15)$, they don't have to hesitate to realize the biological assessment and to repeat it if necessary to confirm the presence of HLH in order to be able to start the specific treatment in time and thus improve the prognosis which remains bleak for this pathology.

The reason for sharing this case is to facilitate knowledge transfer between physicians, caring for adult patients with HLH, with the aim to improve the outcome of these patients.

\section{Competing interests :}

All authors declare no competing interests.

\section{Author contribution:}

Kamal Doghmi: have played a substantial role in designing the article

Mounia Bendari: principal and correspondant author, have played an important role in redaction and literature searching

Nouama Bouanani:co-author, have carried out a revision of the text

Hanane Delsa: participated in writhing

Rajaa Jabbouri : participated in literature searching

Safaa Darouich and Sara Nejjari: have taken aqually part in drafting the article

\section{References:}

1. Buyse S, Teixeira L, Galicier L,Mariotte E, Lemiale V, Seguin A, et al. Critical caremanagement of patients with hemophagocytic lymphohistiocytosis. Intensive Care Med 2010;36(10):1695-702.

2. A Hayden, S Park, D Giustini, A Y.Y. Lee, L Y.C. Chen. Hemophagocytic syndromes (HPSs) including hemophagocytic lymphohistiocytosis (HLH) in adults: A systematic scoping review. Blood Reviews 2016;30(6): 411-420 
3. Risdall RJ, McKenna RW, Nesbit ME et al. Virus-associated hemophagocytic syndrome: a benign histiocytic proliferation distinct from malignant histiocytosis. Cancer 1979;44: 993-1002.

4. O. Lambotte, F. Méchai. Hemophagocytic syndrome. La Lettre de l'Infectiologue - Tome XXII - n 3 - mai-juin 2007

5. zur Stadt U, Schmidt S, Kasper B, Beutel K, Diler AS, Henter JI, et al. Linkage of familial hemophagocytic lymphohistiocytosis (FHL) type-4 to chromosome 6q24 and identification of mutations in syntaxin 11. Hum Mol Genet 2005;14(6):827-34.

6. Emmenegger U, Schaer DJ, Larroche C, Neftel KA. Haemophagocytic syndromes in adults: current concepts and challenges ahead. Swiss Med Wkly 2005;135(21-22):299-314.

7. Ramos-Casals M, Brito-ZerÓn P, LÓpez- Guillermo A, Khamashta MA, Bosch X. Adult haemophagocytic syndrome. Lancet. 2014; 383(9927):1503-1516.

8. Rouphael NG, Talati NJ, Vaughan C, Cunningham K, Moreira R, Gould C. Infections associated with haemophagocytic syndrome. Lancet Infect Dis. 2007;7(12): 814-822.

9. Henter JI, Horne A, AricÓ M, et al. HLH-2004: diagnostic and therapeutic guidelines for hemophagocytic lymphohistiocytosis. Pediatr Blood Cancer. 2007;48(2):124-131.

10. Bergsten E, Horne A, AricÓ M, et al. Confirmed efficacy of etoposide and dexamethasone in HLH treatment: long-term results of the cooperative HLH-2004 study. Blood. 2017;130(25):2728-2738.

11. P. La Rosée, A. Horne, M. Hines, T von Bahr Greenwood, R. Machowicz, N. Berliner, S. Birndt, J. Gil-Herrera. Recommendations for the management of hemophagocytic lymphohistiocytosis in adults. Blood. 2019; 133 (23).

12. V Kollipara, S Hussain, D Franco-Palacios, U Sofi. A case series of endemic infections associated hemophagocytic lymphohistiocytosis (HLH) mimicking severe sepsis syndrome. Respir Med Case Rep. 2019; 27: 100854

13. Machowicz R, Janka G, Wiktor-Jedrzejczak W. Your critical care patient may have HLH (hemophagocytic lymphohistiocytosis). Crit Care. 2016;20(1):215.

14. Mavilia MG, Wu GY. HBV-HCV coinfection: viral interactions, management, and viral reactivation. J Clin Transl Hepatol 2018;6(3):296-305. doi: 10.14218/JCTH.2018.00016.

15. Pol S, Haour G, Fontaine H, Dorival C, Petrov-Sanchez V, Bourliere M, et al. The negative impact of HBV/HCV coinfection on cirrhosis and its consequences. Aliment Pharmacol Ther 2017;46:1054-1060. doi: $10.1111 /$ apt.14352.

16. Yang WT, Wu LW, Tseng TC, Chen CL, Yang HC, Su TH, et al. Hepatitis B surface antigen loss and hepatocellular carcinoma development in patients with dual hepatitis B and C infection. Medicine (Baltimore) 2016;95:e2995. doi: 10.1097/MD.0000000000002995.

17. Calvaruso V, Ferraro D, Licata A, Bavetta MG, Petta S, Bronte F, et al. HBV reactivation in patients with HCV/HBV cirrhosis on treatment with directacting antivirals. J Viral Hepat 2018;25:72-79. doi: 10.1111/jvh.12754.

18. Machowicz R, Janka G, Wiktor-Jedrzejczak W. Similar but not the same: differential diagnosis of HLH and sepsis. Crit Rev Oncol Hematol. 2017; 114:1-12.

19. Jordan MB, Allen CE, Weitzman S, lipovich AH, et coll. How i treat hemophagocytic lymphohistiocytosis. Blood 2011; 118 (15): 4041-4052.

\section{Hosted file}

figures Hemophagocytic lymphohistiocytosis associated with HBV.docx available at https:// authorea.com/users/169778/articles/452088-hemophagocytic-lymphohistiocytosis-associated-

with-hbv-hcv-coinfection-in-adult-case-report 